\title{
Status report on $\varepsilon_{K}$ with lattice QCD inputs
}

\author{
Jon A. Bailey, Weonjong Lee*, Jaehoon Leem, and Sungwoo Park \\ Lattice Gauge Theory Research Center, CTP, and FPRD, \\ Department of Physics and Astronomy, \\ Seoul National University, Seoul 08826, South Korea \\ E-mail: wleeesnu.ac.kr

\section{Yong-Chull Jang} \\ Los Alamos National Laboratory, \\ Theoretical Division T-2, MS B283, \\ Los Alamos, New Mexico 87545, USA \\ E-mail: integration.field@gmail.com
}

\section{SWME Collaboration}

\begin{abstract}
We report the current status of $\varepsilon_{K}$, the indirect $\mathrm{CP}$ violation parameter in the neutral kaon system, evaluated using the lattice QCD inputs. We use lattice QCD to fix $\hat{B}_{K}, \xi_{0}, \xi_{2},\left|V_{u s}\right|, m_{c}\left(m_{c}\right)$, and $\left|V_{c b}\right|$. Since Lattice 2015, FLAG updated $\hat{B}_{K}$, exclusive $V_{c b}$ has been updated with new lattice data in the $\bar{B} \rightarrow D \ell v$ decay channel, and RBC-UKQCD has updated $\xi_{0}$ and $\xi_{2}$. Our preliminary results show that the standard model evaluation of $\varepsilon_{K}$ with exclusive $\left|V_{c b}\right|$ (lattice QCD inputs) has $3.2 \sigma$ tension with the experimental value, while that of $\varepsilon_{K}$ with inclusive $\left|V_{c b}\right|$ (heavy quark expansion) shows no tension.
\end{abstract}

34th annual International Symposium on Lattice Field Theory

24-30 July 2016

University of Southampton, UK

\footnotetext{
* Speaker.
} 


\section{Introduction}

This paper is a follow-up and update of our previous paper [1,2]. In the standard model, the indirect $\mathrm{CP}$ violation parameter of the neutral kaon system $\varepsilon_{K}$ is

$$
\begin{aligned}
\varepsilon_{K} & \equiv \frac{\mathscr{A}\left(K_{L} \rightarrow \pi \pi(I=0)\right)}{\mathscr{A}\left(K_{S} \rightarrow \pi \pi(I=0)\right)} \\
& =e^{i \theta} \sqrt{2} \sin \theta\left(C_{\varepsilon} \hat{B}_{K} X_{\mathrm{SD}}+\frac{\xi_{0}}{\sqrt{2}}+\xi_{\mathrm{LD}}\right)+\mathscr{O}\left(\omega \varepsilon^{\prime}\right)+\mathscr{O}\left(\xi_{0} \Gamma_{2} / \Gamma_{1}\right),
\end{aligned}
$$

where $C_{\varepsilon}$ is a well-known coupling, and $X_{\mathrm{SD}}$ is the short distance contribution from the box diagrams. Master formulas for $C_{\varepsilon}, X_{\mathrm{SD}}, \xi_{0}$, and $\xi_{\mathrm{LD}}$ are given in Ref. [1].

Since Lattice 2015, there have been major updates of lattice QCD inputs such as $V_{c b}, \hat{B}_{K}, \xi_{0}$, and $\xi_{2}$. Hence, it is time to update the current status of $\varepsilon_{K}$.

\section{Input parameter $\left|V_{c b}\right|$}

\begin{tabular}{lll}
\hline \hline Decay mode & $\left|V_{c b}\right|$ & Ref. \\
\hline $\bar{B} \rightarrow D^{*} \ell \bar{v}$ & $39.04(49)(53)(19)$ & {$[3]$} \\
\hline $\bar{B} \rightarrow D \ell \bar{v}$ & $40.7(10)(2)$ & {$[4]$} \\
\hline ex-combined & $39.62(60)$ & this paper \\
\hline $\bar{B} \rightarrow X_{c} \ell \bar{v}$ & $42.00(64)$ & {$[5]$} \\
\hline \hline Decay mode & $\left|V_{u b}\right|$ & Ref. \\
\hline $\bar{B} \rightarrow \pi \ell \bar{v}$ & $3.70(14)$ & {$[6,7]$} \\
\hline $\bar{B} \rightarrow X_{u} \ell \bar{v}$ & $4.45(16)(22)$ & {$[8]$} \\
\hline \hline Decay mode & $\left|V_{u b} / V_{c b}\right|$ & Ref. \\
\hline$\Lambda_{b} \rightarrow \Lambda_{c} \ell \bar{v}$ & $0.083(4)(4)$ & {$[9]$} \\
\hline \hline
\end{tabular}

Table 1: Results of $\left|V_{c b}\right|$ and $\left|V_{u b}\right|$.

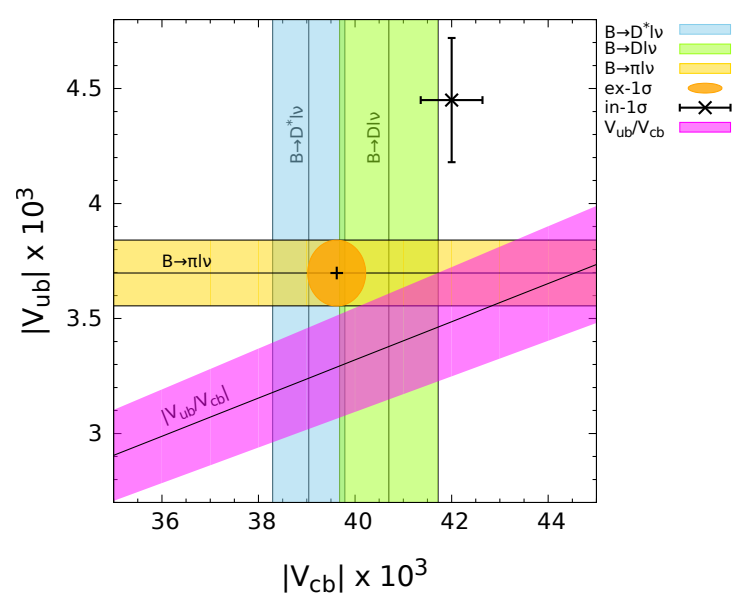

Figure 1: $\left|V_{c b}\right|$ versus $\left|V_{u b}\right|$.

Let us begin with $V_{c b}$. In Table 1, we summarize updated results for $\left|V_{c b}\right|$ and $\left|V_{u b}\right|$. In Ref. [4], DeTar has collected the results for the $\bar{B} \rightarrow D \ell \bar{v}$ decay mode at non-zero recoil from both lattice QCD [10,11] and the experiments of Babar [12] and Belle [13] to make a combined fit of all of them. This result corresponds to the green band in Fig. 1. We combine the results of Refs. [4] $(\bar{B} \rightarrow D \ell \bar{v})$ and $[3]\left(\bar{B} \rightarrow D^{*} \ell \bar{v}\right)$ to obtain the uncorrelated weighted average, which corresponds to the "ex-combined" result in Table 1. This value is shown as an orange circle in Fig. 1. The black cross represents results of inclusive $\left|V_{c b}\right|$ and $\left|V_{u b}\right|$. The inclusive results are about $3 \sigma$ away from those of the exclusive decays as well as the LHCb results of $\left|V_{u b} / V_{c b}\right|$ (the magenta band in Fig. 1).

\section{Input parameter $\xi_{0}$}

There are two independent methods to determine $\xi_{0}$ in lattice QCD: One is the indirect method, 


\begin{tabular}{llll}
\hline \hline Input & Method & Value & Ref. \\
\hline$\xi_{0}$ & indirect & $-1.63(19) \times 10^{-4}$ & {$[14]$} \\
\hline$\xi_{0}$ & direct & $-0.57(49) \times 10^{-4}$ & {$[15]$} \\
\hline$\xi_{\mathrm{LD}}$ & - & $(0 \pm 1.6) \%$ & {$[16]$} \\
\hline \hline
\end{tabular}

(a) Long Distance Effects

\begin{tabular}{lll}
\hline Collaboration & Value & Ref. \\
\hline FLAG-2016 & $0.7625(97)$ & {$[17]$} \\
\hline SWME-2014 & $0.7379(47)(365)$ & {$[18]$} \\
\hline RBC-UK-2016 & $0.7499(24)(150)$ & {$[19]$} \\
\hline \hline
\end{tabular}

(b) $\hat{B}_{K}$

Table 2: Input parameters: $\xi_{0}, \xi_{\mathrm{LD}}$ and $\hat{B}_{K}$

and the other is the direct method. The parameter $\xi_{0}$ is connected with $\varepsilon^{\prime} / \varepsilon$ and $\xi_{2}$ as follows,

$$
\xi_{0}=\frac{\operatorname{Im} A_{0}}{\operatorname{Re} A_{0}}, \quad \xi_{2}=\frac{\operatorname{Im} A_{2}}{\operatorname{Re} A_{2}}, \quad \operatorname{Re}\left(\frac{\varepsilon^{\prime}}{\varepsilon}\right)=\frac{\omega}{\sqrt{2}\left|\varepsilon_{K}\right|}\left(\xi_{2}-\xi_{0}\right) .
$$

In the indirect method, we determine $\xi_{0}$ from the experimental values of $\operatorname{Re}\left(\varepsilon^{\prime} / \varepsilon\right), \varepsilon_{K}, \omega$, and the lattice QCD input $\xi_{2}$ using Eq. (3.1). Recently, RBC-UKQCD reported new results for $\xi_{2}$ in Ref. [14]. The results for $\xi_{0}$ using the indirect method are summarized in Table 2(a).

Recently, RBC-UKQCD also reported new lattice QCD results for $\operatorname{Im} A_{0}$ calculated using domain wall fermions [15]. Using the experimental value of $\operatorname{Re} A_{0}$, we can determine $\xi_{0}$ directly from $\operatorname{Im} A_{0}$. RBC-UKQCD also reported the S-wave $\pi-\pi$ (I=0) scattering phase shift $\delta_{0}=23.8(49)(12)$ [15]. This value is $3.0 \sigma$ lower than the conventional determination of $\delta_{0}$ in Refs. [20] (KPY-2011) and [21, 22] (CGL-2001). The values for $\delta_{0}$ are summarized in Table 3. In Fig. 2, we show the results of KPY-2011. They used a singly subtracted Roy-like equation to do the interpolation around $\sqrt{s}=m_{K}$ (kaon mass). Their fitting to the experimental data works well from the threshold to $\sqrt{s}=800 \mathrm{MeV}$.

\begin{tabular}{lll}
\hline \hline Collaboration & $\delta_{0}$ & Ref. \\
\hline RBC-UK-2016 & $23.8(49)(12)^{\circ}$ & {$[15]$} \\
\hline KPY-2011 & $39.1(6)^{\circ}$ & {$[20]$} \\
\hline CGL-2001 & $39.2(15)^{\circ}$ & {$[21,22]$} \\
\hline \hline
\end{tabular}

Table 3: Results of $\delta_{0}$

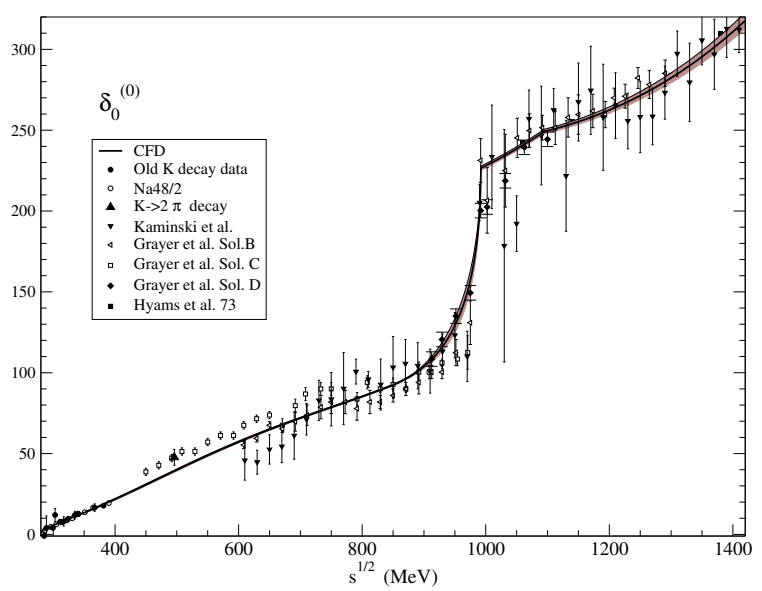

Figure 2: Experimental results of $\delta_{0}$

In Fig. 3(a), we show the fitting results of both KPY-2011 and CGL-2001 as well as the RBCUKQCD result. There is essentially no difference between KPY-2011 and CGL-2001 in the region near $\sqrt{s}=m_{K}$. Here, we observe the $3.0 \sigma$ gap between RBC-UKQCD and KPY-2011. In contrast, in the case of $\delta_{2}$ (S-wave, I=2), there is no difference between RBC-UKQCD and KPY-2011 within statistical uncertainty. 


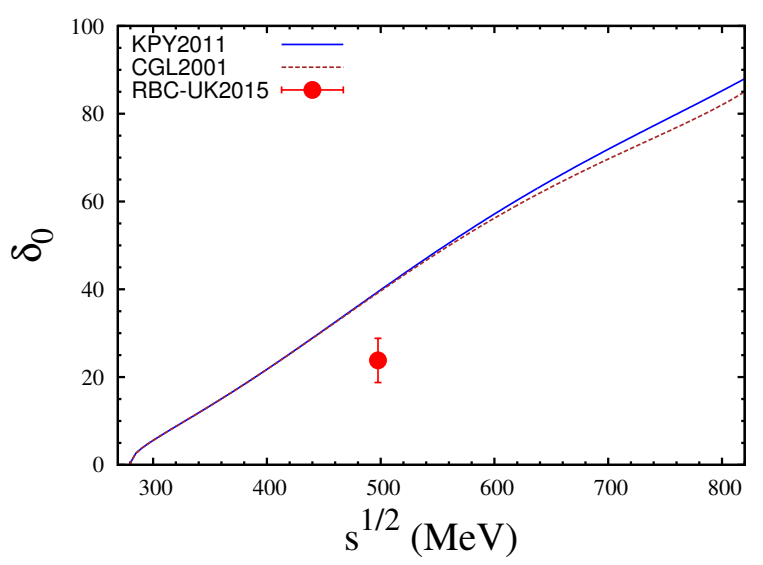

(a) $\delta_{0}$

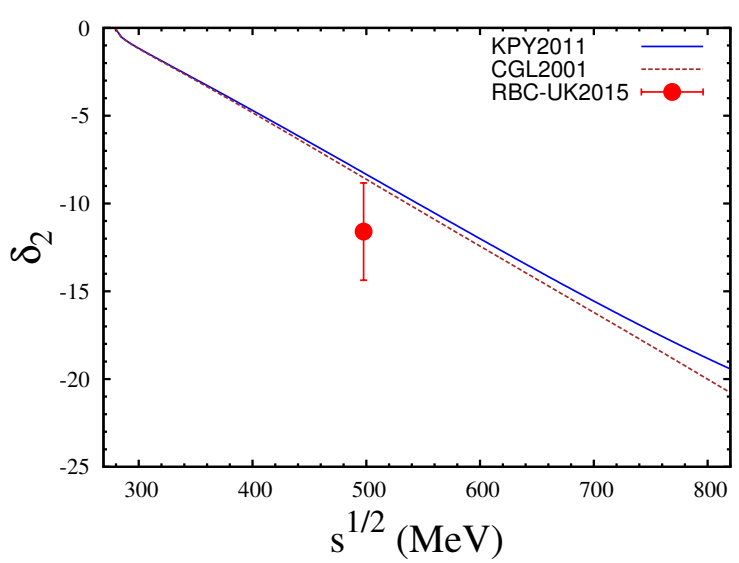

(b) $\delta_{2}$

Figure 3: S-wave $\pi-\pi$ scattering phase shifts with $I=0$ and $I=2$.

Considering all aspects, we conclude that the direct calculation of $\operatorname{Im} A_{0}$ and $\xi_{0}$ by RBCUKQCD in Ref. [15] may have unresolved issues. Hence, we use the indirect method to determine $\xi_{0}$ in this paper.

Regarding $\xi_{\mathrm{LD}}$, the long distance effect in the dispersive part, there has been an on-going attempt to calculate it on the lattice [23]. However, this attempt [24], at present, belongs to the category of exploratory study rather than to that of precision measurement. Hence, we use the rough estimate of $\xi_{\mathrm{LD}}$ in Ref. [23] in this paper, which is given in Table 2(a).

\section{Input parameter $\hat{B}_{K}$}

In Table 2(b), we present results for $\hat{B}_{K}$ calculated in lattice QCD with $N_{f}=2+1$ flavors. Here, FLAG-2016 represents the global average over the results of BMW-2011 [25], Laiho-2011 [26], RBC-UK-2016 [19], and SWME-2016 [27], which is reported in Ref. [17]. SWME-2014 represents the $\hat{B}_{K}$ result reported in Ref. [18]. RBC-UK-2016 represents that reported in Ref. [19].

The results of SWME-2016 are obtained using fitting based on staggered chiral perturbation theory (SChPT) in the infinite volume limit, while those of SWME-2014 are obtained using fitting based on SChPT with finite volume corrections included at the NLO level. In this paper, we use the FLAG-2016 result of $\hat{B}_{K}$.

\section{Other input parameters}

For the Wolfenstein parameters $\lambda, \bar{\rho}$, and $\bar{\eta}$, both CKMfitter and UTfit updated their results in Refs. [28, 29], while the angle-only-fit has not been updated since 2015. The results are summarized in Table 4(a).

For the QCD corrections $\eta_{c c}, \eta_{c t}$, and $\eta_{t t}$, we use the same values as in Ref. [1], which are given in Table 4(b). Other input parameters are the same as in Ref. [1] except for the charm quark mass $m_{c}\left(m_{c}\right)$, which are summarized in Table 4(c). For the charm quark mass, we use the HPQCD results of $m_{c}\left(m_{c}\right)$ reported in Ref. [30]. 


\begin{tabular}{cccc}
\hline \hline & CKMfitter & UTfit & AOF [31] \\
\hline$\lambda$ & $0.22548(68) /[28]$ & $0.22497(69) /[29]$ & $0.2253(8) /[32]$ \\
\hline $\bar{\rho}$ & $0.145(13) /[28]$ & $0.153(13) /[29]$ & $0.139(29) /[33]$ \\
\hline $\bar{\eta}$ & $0.343(12) /[28]$ & $0.343(11) /[29]$ & $0.337(16) /[33]$ \\
\hline \hline
\end{tabular}

(a) Wolfenstein parameters

\begin{tabular}{clc}
\hline \hline Input & Value & Ref. \\
\hline$\eta_{c c}$ & $1.72(27)$ & {$[1]$} \\
\hline$\eta_{t t}$ & $0.5765(65)$ & {$[34]$} \\
\hline$\eta_{c t}$ & $0.496(47)$ & {$[35]$} \\
\hline \hline
\end{tabular}

(b) QCD corrections

\begin{tabular}{clc}
\hline \hline Input & Value & Ref. \\
\hline$G_{F}$ & $1.1663787(6) \times 10^{-5} \mathrm{GeV}^{-2}$ & {$[32]$} \\
\hline$M_{W}$ & $80.385(15) \mathrm{GeV}$ & {$[32]$} \\
\hline$m_{c}\left(m_{c}\right)$ & $1.2733(76) \mathrm{GeV}$ & {$[30]$} \\
\hline$m_{t}\left(m_{t}\right)$ & $163.3(2.7) \mathrm{GeV}$ & {$[36]$} \\
\hline$\theta$ & $43.52(5)^{\circ}$ & {$[32]$} \\
\hline$m_{K^{0}}$ & $497.614(24) \mathrm{MeV}$ & {$[32]$} \\
\hline$\Delta M_{K}$ & $3.484(6) \times 10^{-12} \mathrm{MeV}$ & {$[32]$} \\
\hline$F_{K}$ & $156.2(7) \mathrm{MeV}$ & {$[32]$} \\
\hline \hline
\end{tabular}

(c) Other input parameters

Table 4: Input parameters

\section{Results for $\varepsilon_{K}$ with lattice QCD inputs}

In Fig. 4, we show the results for $\varepsilon_{K}$ evaluated directly from the standard model with the lattice QCD inputs described in the previous sections. In Fig. 4(a), the blue curve represents the theoretical evaluation of $\varepsilon_{K}$ with the FLAG $\hat{B}_{K}$, AOF for the Wolfenstein parameters, and exclusive $V_{c b}$ that corresponds to ex-combined in Table 1 . Here the red curve represents the experimental value of $\varepsilon_{K}$. In Fig. 4(b), the blue curve represents the same as in 4(a) except for using the inclusive $V_{c b}$ in Table 1. Our preliminary results are, in units of $1.0 \times 10^{-3}$,

$$
\begin{aligned}
& \left|\varepsilon_{K}\right|=1.69 \pm 0.17 \quad \text { for exclusive } V_{c b} \text { (lattice QCD) } \\
& \left|\varepsilon_{K}\right|=2.10 \pm 0.21 \quad \text { for inclusive } V_{c b} \text { (QCD sum rules) } \\
& \left|\varepsilon_{K}\right|=2.228 \pm 0.011 \quad \text { (experimental value) }
\end{aligned}
$$

This indicates that there is $3.2 \sigma$ tension in the exclusive $V_{c b}$ channel (lattice QCD) and no tension in the inclusive $V_{c b}$ channel (heavy quark expansion; QCD sum rules).

\section{Acknowledgments}

We thank R. Van de Water for helpful discussion on $V_{c b}$. The research of W. Lee is supported by the Creative Research Initiatives Program (No. 20160004939) of the NRF grant funded by the Korean government (MEST). J.A.B. is supported by the Basic Science Research Program of the National Research Foundation of Korea (NRF) funded by the Ministry of Education (No. 2015024974). W. Lee would like to acknowledge the support from the KISTI supercomputing center through the strategic support program for the supercomputing application research 


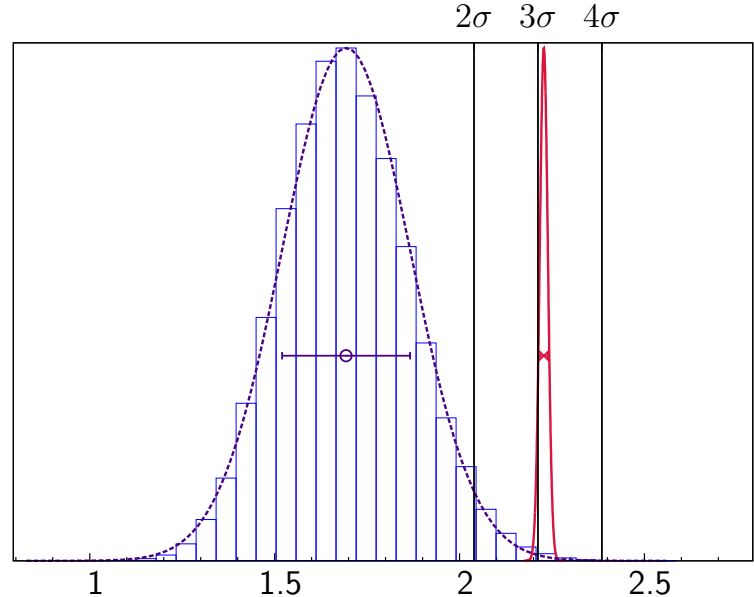

(a) $\varepsilon_{K}$ with exclusive $V_{c b}$

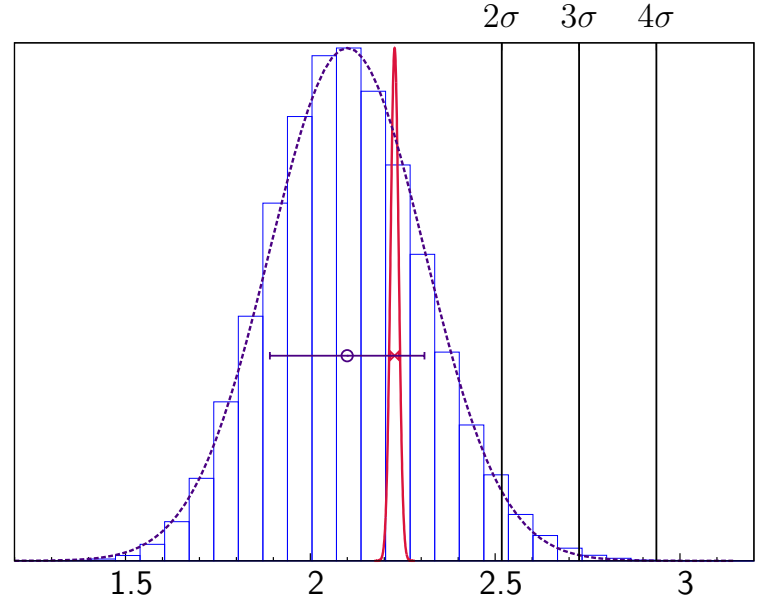

(b) $\varepsilon_{K}$ with inclusive $V_{c b}$

Figure 4: $\varepsilon_{K}$ with exclusive $V_{c b}$ (left) and inclusive $V_{c b}$ (right). Here, we use the FLAG-2016 $\hat{B}_{K}$ and $\mathrm{AOF}$ for the Wolfenstein parameters. The red curve represents the experimental value of $\varepsilon_{K}$ and the blue curve the theoretical value evaluated directly from the standard model.

(No. KSC-2014-G3-003). Computations were carried out on the DAVID GPU clusters at Seoul National University.

\section{References}

[1] SWME Collaboration, J. A. Bailey, Y.-C. Jang, W. Lee, and S. Park Phys. Rev. D92 (2015), no. 3 034510, [1503.05388].

[2] J. A. Bailey, Y.-C. Jang, W. Lee, and S. Park PoS LATTICE2015 (2015) 348, [1511. 00969 ].

[3] J. A. Bailey, A. Bazavov, C. Bernard, et al. Phys.Rev. D89 (2014) 114504, [1 403.0635 ].

[4] C. DeTar in Proceedings of the 27th International Symposium on Lepton Photon Interactions at High Energies, 2015. 1511.06884.

[5] P. Gambino, K. J. Healey, and S. Turczyk 1606.06174.

[6] Fermilab Lattice, MILC Collaboration, J. A. Bailey et al. Phys. Rev. D92 (2015), no. 1014024 , [1503.07839].

[7] J. M. Flynn, T. Izubuchi, T. Kawanai, C. Lehner, A. Soni, R. S. Van de Water, and O. Witzel Phys. Rev. D91 (2015), no. 7 074510, [1501. 05373].

[8] Heavy Flavor Averaging Group (HFAG) Collaboration, Y. Amhis et al. 1412.7515.

[9] W. Detmold, C. Lehner, and S. Meinel Phys. Rev. D92 (2015), no. 3 034503, [1503. 01421 ].

[10] MILC Collaboration, J. A. Bailey et al. Phys. Rev. D92 (2015), no. 3 034506, [1503. 07237].

[11] HPQCD Collaboration, H. Na, C. M. Bouchard, G. P. Lepage, C. Monahan, and J. Shigemitsu Phys. Rev. D92 (2015), no. 5 054510, [1505. 03925]. [Erratum: Phys. Rev.D93,no.11,119906(2016)].

[12] BaBar Collaboration, B. Aubert et al. Phys. Rev. D79 (2009) 012002, [0 809 . 0828].

[13] R. Glattauer PoS EPS-HEP2015 (2015) 554. 
[14] T. Blum et al. Phys. Rev. D91 (2015), no. 7 074502, [1502.00263].

[15] RBC, UKQCD Collaboration, Z. Bai et al. Phys. Rev. Lett. 115 (2015), no. 21212001 , [1505.07863].

[16] N. Christ, T. Izubuchi, C. Sachrajda, A. Soni, and J. Yu Phys.Rev. D88 (2013), no. 1 014508, [1212.5931].

[17] S. Aoki et al., Review of lattice results concerning low-energy particle physics, 1607.00299.

[18] T. Bae et al. Phys.Rev. D89 (2014) 074504, [1402 . 0048$].$

[19] RBC, UKQCD Collaboration, T. Blum et al. Phys. Rev. D93 (2016), no. 7 074505, [1411. 7017 ].

[20] R. Garcia-Martin, R. Kaminski, J. R. Pelaez, J. Ruiz de Elvira, and F. J. Yndurain Phys. Rev. D83 (2011) 074004, [1102.2183].

[21] G. Colangelo, J. Gasser, and H. Leutwyler Nucl. Phys. B603 (2001) 125-179, [hep-ph/ 0103088 ].

[22] https://indico.mitp.uni-mainz.de/event/48/contribution/5/material/ slides/0.pdf.

[23] N. Christ, T. Izubuchi, C. T. Sachrajda, A. Soni, and J. Yu PoS LATTICE2013 (2014) 397, [1402.2577].

[24] https://conference.ippp.dur.ac.uk/event/470/session/15/contribution/407/material/slides/0.pdf.

[25] S. Durr, Z. Fodor, C. Hoelbling, et al. Phys.Lett. B705 (2011) 477-481, [1106.3230].

[26] J. Laiho and R. S. Van de Water PoS LATTICE2011 (2011) 293, [1112 . 4861].

[27] SWME Collaboration, B. J. Choi et al., Kaon BSM B-parameters using improved staggered fermions from $N_{f}=2+1$ unquenched QCD, Phys. Rev. D93 (2016), no. 1 014511, [1509.00592].

[28] J. Charles et al. Phys. Rev. D91 (2015), no. 7 073007, [1501.05013].

[29] http://www.utfit.org/UTfit/ResultsSummer2016SM.

[30] B. Chakraborty, C. T. H. Davies, B. Galloway, P. Knecht, J. Koponen, G. C. Donald, R. J. Dowdall, G. P. Lepage, and C. McNeile Phys. Rev. D91 (2015), no. 5 054508, [1408.4169].

[31] A. Bevan, M. Bona, M. Ciuchini, D. Derkach, E. Franco, et al. Nucl.Phys.Proc.Suppl. 241-242 (2013) 89-94.

[32] K. Olive et al. Chin.Phys. C38 (2014) 090001.

[33] http://www.utfit.org/UTfit/ResultsSummer2014PostMoriondSM.

[34] A. J. Buras and D. Guadagnoli Phys.Rev. D78 (2008) 033005, [0 805 . 3887].

[35] J. Brod and M. Gorbahn Phys.Rev. D82 (2010) 094026, [1 007.068 ]].

[36] S. Alekhin, A. Djouadi, and S. Moch Phys.Lett. B716 (2012) 214-219, [1207 . 0980 ]. 